\title{
Development of an animal model of nephrocalcinosis via selective dietary sodium and chloride depletion
}

\author{
Shamir Tuchman ${ }^{1,2}$, Laureano D. Asico ${ }^{2}$, Crisanto Escano ${ }^{2}$, Daniel A. Bobb ${ }^{3}$ and Patricio E. Ray ${ }^{1,2}$
}

\begin{abstract}
BACKGROUND: Nephrocalcinosis (NC) is an important clinical problem seen in critically ill preterm neonates treated with loop diuretics. No reliable animal models are available to study the pathogenesis of NC in preterm infants. The purpose of this study was to develop a reproducible and clinically relevant animal model of $\mathrm{NC}$ for these patients and to explore the impact of extracellular fluid (ECF) volume contraction induced by sodium and chloride depletion in this process.
\end{abstract}

METHODS: Three-week-old weanling Sprague-Dawley rats were fed diets deficient in either chloride or sodium or both. A subgroup of rats from each dietary group was injected daily with furosemide ( $40 \mathrm{mg} / \mathrm{kg}$ i.p.).

RESULTS: Rats fed a control diet, with or without furosemide, or a chloride-depleted diet alone, did not develop NC. By contrast, $50 \%$ of the rats injected with furosemide and fed the chloride-depleted diet developed NC. Moreover, $94 \%$ of the rats fed the combined sodium- and chloride-depleted diet developed NC, independently of furosemide use. NC was associated with the development of severe ECF volume contraction; hypochloremic, hypokalemic, metabolic alkalosis; increased phosphaturia; and growth retardation.

CONCLUSION: Severe ECF volume contraction induced by chronic sodium and chloride depletion appears to play an important role in the pathogenesis of NC.

N ephrocalcinosis (NC) is a well-described complication seen frequently in preterm infants and in older children with inherited and acquired renal tubulopathies. Furthermore, NC is a well-described complication of congenital chloride diarrhea (CLD), which, with time, contributes to the renal injury observed in these patients (1). The pathogenesis of NC in preterm neonates has been associated with the use of chronic loop diuretics, as well as with the presence of electrolyte changes or critical illness $(2,3)$. However, the basic pathogenic mechanisms involved in NC are not clearly understood, and there has been no definitive link between the development of NC in preterm neonates and the duration or dose of loop diuretic therapy. Estimates of the incidence of $\mathrm{NC}$ on the basis of screening of preterm infants range from $7 \%$ to $39 \%(4-7)$. NC has been associated with the development of renal insufficiency and hypertension in preterm neonates treated with furosemide, in infants with inherited renal tubulopathies, and in children with CLD (8-11). Hypercalciuria (HC) has not been a necessarily consistent finding in all infants with NC $(12,13)$ and is also not typically present in children with CLD (8), which speaks to the nonessential role of HC in the pathogenesis of this disease. NC does not uniformly resolve in infants who are taken off loop diuretic therapy and certainly persists in children with inherited tubulopathies and CLD $(9,14,15)$. Furthermore, the ability of loop diuretics to promote $\mathrm{NC}$ in preterm infants does not seem to result from an increase in stone-promoting substances such as oxalate (16).

Currently, no animal models exist that attempt to replicate the fluid and electrolyte abnormalities that are associated with the development of $\mathrm{NC}$ in the preterm infant. A previous model using i.p. furosemide alone in weanling rats on a control diet was able to induce NC (17); however, these changes could not be reproduced in our preliminary experiments. In addition, furosemide has not been shown to induce NC in adult rats fed control diets (18).

Other studies in adult rats have produced NC using a combination of chloride depletion with either phosphate loading or highsulfate diet $(18,19)$. Isolated phosphate loading has consistently produced NC only in young rats (20). Therefore, the purpose of the current study was to develop a reliable and reproducible animal model system of NC that mimics the pathophysiological alterations associated with the development of NC in preterm neonates receiving furosemide, children with inherited renal tubulopathies, or children with extrarenal losses of volume and electrolytes such as those that occur in CLD or pyloric stenosis.

\section{RESULTS}

Chloride- and Sodium-Deficient Diets Induced NC in Young Rats The most remarkable finding was that almost all rats that were fed the combined sodium and chloride-deficient diet developed moderate to severe NC ( $P$ value $<0.001$ as compared with controls) (Table 1). Representative histologic samples from these groups and controls are shown in Figure 1. Young rats treated with furosemide alone, or fed a selective chloride-deficient diet alone, developed hypokalemic, hypochloremic, metabolic alkalosis, but these changes were not sufficient to induce NC (Table 1). However, 50\% of the chloride-depleted rats treated with i.p. furosemide developed moderate to severe NC

'Division of Pediatric Nephrology, Children's Research Institute, Children's National Medical Center, Washington, DC; ${ }^{2}$ Center for Molecular Physiology, Children's Research Institute, Children's National Medical Center, Washington, DC; ${ }^{3}$ Research Animal Facility, Children's Research Institute, Children's National Medical Center, Washington, DC. Correspondence: Shamir Tuchman (stuchman@cnmc.org) 


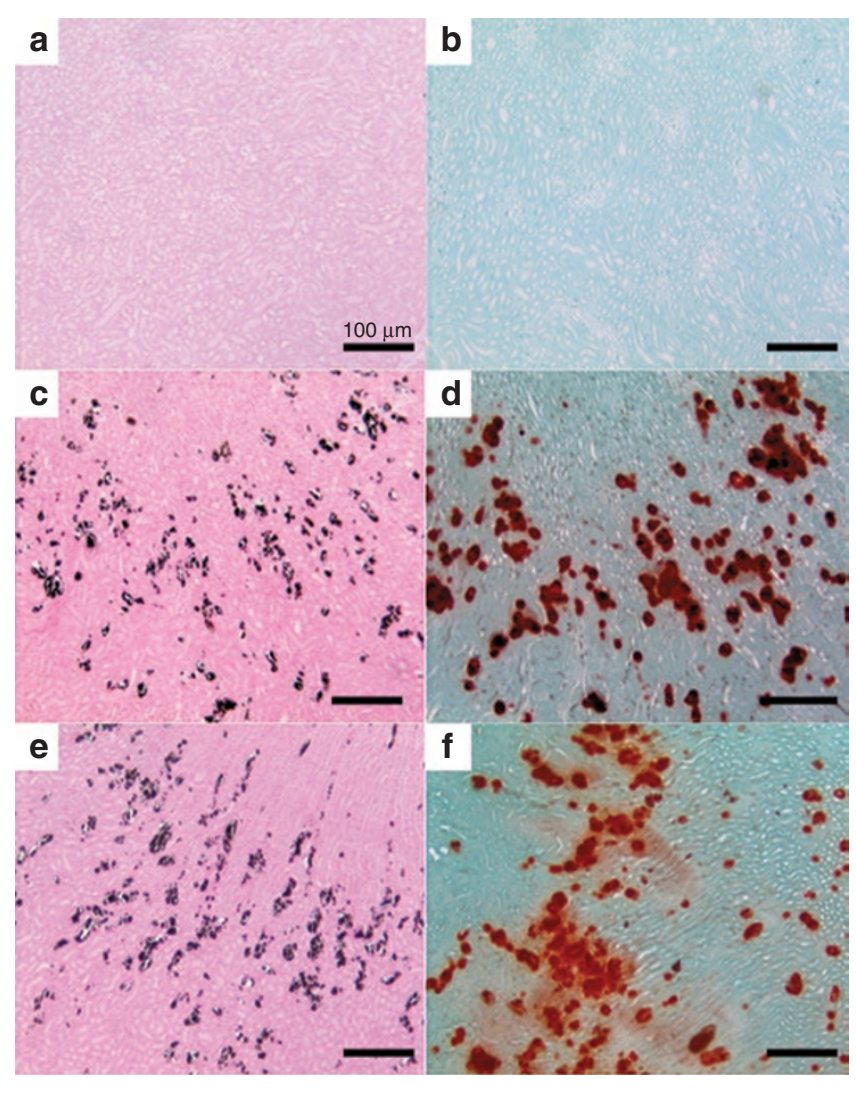

Figure 1. von Kossa and Alizarin red stains of kidney sections from Sprague-Dawley rats. The panel depicts (a) von Kossa and (b) Alizarin red staining from rats fed a control diet; (c) von Kossa and (d) Alizarin red staining from rats fed a low chloride diet + i.p. furosemide; and (e) von Kossa and (f) Alizarin red staining from rats fed a low-sodium and lowchloride diet. All panels represent $\times 25$ original magnification.

Table 1. Occurrence of NC by dietary intervention group

\begin{tabular}{lcccc}
\hline & $n$ & $\begin{array}{c}\text { Mild NC } \\
\text { (category 1) }\end{array}$ & $\begin{array}{c}\text { Moderate NC } \\
\text { (category 2-3) }\end{array}$ & $\begin{array}{c}\text { Severe NC } \\
\text { (category 4-5) }\end{array}$ \\
\hline Control & 7 & 0 & 0 & 0 \\
$\quad$ With/furosemide) & 6 & 0 & 0 & 0 \\
Low chloride & 11 & 0 & 0 & 0 \\
$\quad$ With/furosemide) & 12 & $1(8 \%)$ & $3(25 \%)$ & $2(17 \%)$ \\
$\begin{array}{l}\text { Low sodium/ } \\
\text { chloride }\end{array}$ & 18 & 0 & $3(17 \%)$ & $14(78 \%)$ \\
$\quad$ With/furosemide) & 12 & 0 & $3(25 \%)$ & $9(75 \%)$ \\
\hline NC, nephrocalcinosis. & & & &
\end{tabular}

$N C$, nephrocalcinosis.

$(P$ value $<0.01)$. These changes were also associated with the development of hyponatremia and decreased urinary sodium excretion (Table 2). In general, animals that developed more severe metabolic alkalosis (e.g., higher serum bicarbonate concentration) also showed lower levels of ionized calcium.

Examination of rat kidney sections stained with von Kossa and hematoxylin and eosin (data not shown) under polarized light failed to reveal birefringent crystals in animals with NC. This, as also confirmed on Alizaren red stains (Figure 1), shows that most deposits were composed primarily of calcium phosphate. None of rats that were fed a control diet developed NC.

\section{NC Induced Through Severe ECF Volume Contraction}

Rats that developed severe extracellular fluid (ECF) volume contraction, as evidenced by decreased weight, increased hematocrit, and increased blood urea nitrogen, were more likely to develop NC (Table 3). However, selective chloridedeficient rats developed significant ECF volume contraction but did not develop NC. These findings suggest that the ECF volume contraction per se is not sufficient to induce NC. Rats fed a low-sodium/chloride diet had a higher mean urine output $(\mathrm{ml} / \mathrm{d})(P<0.05)$ than rats fed a control diet (Table 3$)$. In the context of ECF volume contraction, this represents inappropriate urinary fluid losses. Rats that were administered i.p. furosemide with either a low-chloride or low-sodium and lowchloride diet did not have a higher daily urine output than rats that were given the respective diets alone (Table 3 ).

\section{Urine Calcium Excretion in Rats With NC}

As shown in Figure 2, rats from dietary intervention groups (e.g., low-chloride diet + furosemide or low-sodium and lowchloride diet alone) that developed NC had mean urine calcium to creatinine ratios that were not different from those in animals fed a control diet. Across all dietary intervention groups, rats that developed $\mathrm{NC}$ had a significantly lower urine calcium to creatinine excretion ratio $(0.22$ vs. $0.31 \mathrm{mg} / \mathrm{mg}$ creatinine, $P<0.05)$ than those that did not. This relationship also held true within the group of rats fed a low-chloride diet and given i.p. furosemide in which $50 \%$ of animals developed NC. Among this group, urine calcium excretion was significantly lower in rats that developed NC ( 0.18 vs. $0.29 \mathrm{mg} /$ $\mathrm{mg}$ creatinine, $P<0.05)$. Across all study groups, the ionized calcium (mean $\pm \mathrm{SD}$ ) was $1.13 \pm 0.11$ among rats with $\mathrm{NC}$ and $1.26 \pm 0.12$ in rats without $\mathrm{NC}(P<0.001)$. A decreased whole-blood ionized calcium level was associated with a hypochloremic, hypokalemic, metabolic alkalosis in all rats (Table 2). In a logistic regression model examining urinary parameters associated with $\mathrm{NC}$, a lower urinary potassium excretion ( $\beta$-coefficient $(\beta)=-3.8, P<0.01)$ and a higher 24 -h urine output ( $\beta=0.02, P=0.05$ ) were also associated with the development of NC.

\section{Association of Urine Phosphorus Excretion With NC}

As shown in Table 2, urinary phosphorus excretion was more than twofold higher among rats fed a low-sodium and lowchloride diet as compared with controls. In addition, among rats fed a low-chloride diet and were administered i.p. furosemide, those that did develop NC had a 10-fold higher urinary phosphorus excretion than those that did not $(P<0.05)$. The lowest urine phosphorus excretion was seen among rats fed a low-chloride diet alone, speaking against the notion that increased urine phosphorus excretion resulted directly from chloride depletion. Urine magnesium excretion did not show a definitive association with NC despite the fact that rats fed a control diet had the lowest excretion (Table 2). The highest 
Table 2. Lab parameters (M (SD)) in Sprague-Dawley rats by dietary intervention group

\begin{tabular}{|c|c|c|c|c|c|c|}
\hline & Control & $\begin{array}{l}\text { Control + } \\
\text { furosemide }\end{array}$ & $\begin{array}{l}\text { Low } \\
\mathrm{Cl}^{-}\end{array}$ & $\begin{array}{c}\text { Low } \mathrm{Cl}^{-} \\
\text {furosemide }\end{array}$ & $\begin{array}{c}\text { Low } \\
\mathrm{Na}^{+} / \mathrm{Cl}^{-}\end{array}$ & $\begin{array}{l}\text { Low } \mathrm{Na}^{+} / \mathrm{Cl}^{-} \\
\text {furosemide }\end{array}$ \\
\hline$N$ & 7 & 6 & 11 & 12 & 18 & 12 \\
\hline $\mathrm{Na}^{+}(\mathrm{mmol} / \mathrm{l})$ & $137.3(0.5)$ & $135.6(1.1)^{*, * *, * * *, \dagger}$ & $118.1(2.9)^{*}$ & $113.9(4.3)^{*, * *}$ & $116.3(4.7)^{*}$ & $122.2(4.4)^{*, * *, * * *, \dagger}$ \\
\hline $\mathrm{K}^{+}(\mathrm{mmol} / \mathrm{l})$ & $3.7(0.2)$ & $3.1(0.3)^{*, * * *,+}$ & $2.7(0.5)^{*}$ & $2.2(0.2)^{*, * *}$ & $2.7(0.4)^{*,+}$ & $2.7(0.3)^{*,+}$ \\
\hline $\mathrm{Na}^{+}(\mathrm{mmol} / \mathrm{d})$ & $0.43(0.17)$ & $0.78(0.01)^{*, * * * * * *, \dagger}$ & $0.17(0.11)^{*}$ & $0.23(0.11)^{*}$ & $0.14(0.11)^{*,+}$ & $0.25(0.2)$ \\
\hline $\mathrm{K}^{+}(\mathrm{mmol} / \mathrm{d})$ & $0.48(0.16)$ & $0.67(0.22)$ & $1.07(0.51)^{*}$ & $0.88(0.34)^{*}$ & $0.60(0.20)^{* *,+}$ & $0.50(0.17)^{* *,+}$ \\
\hline $\mathrm{Cl}^{-}(\mathrm{mmol} / \mathrm{d})$ & $0.54(0.16)$ & $1.56(0.19)^{*, * * * * * *, \dagger}$ & $0.31(0.19)^{*}$ & $0.39(0.21)$ & $0.19(0.20)^{*,+}$ & $0.29(0.25)^{*}$ \\
\hline Creatinine (mg/dl) & $53.6(17.9)$ & $21.1(15.6)^{*}$ & $23.5(13.4)^{*}$ & $12(2.4)^{*, * *}$ & $15.3(1.6)^{*, * *,+}$ & $11.1(2.9)^{*, * *, \dagger}$ \\
\hline $\mathrm{pH}$ & $7.44(0.04)$ & $7.50(0.05)^{*, * * * * * *,+}$ & $7.62(0.13)^{*}$ & $7.64(0.03)^{*}$ & $7.58(0.05)^{*, * *,+}$ & $7.53(0.14)^{\dagger}$ \\
\hline $\mathrm{HCO}_{3}(\mathrm{mg} / \mathrm{dl})$ & $30.3(2.3)$ & $32.4(2.6)^{* * * * * *,+}$ & $41.1(4.9)^{*}$ & $51.5(8.4)^{* * * * *}$ & $45.0(4.6)^{*, * *,+}$ & $38.7(4.5)^{*, * * *,+}$ \\
\hline Ionized $\mathrm{Ca}^{2+}$ & $1.33(0.04)$ & $1.35(0.1)^{* *, * * *, \dagger}$ & $1.15(0.03)^{*}$ & $1.03(0.07)^{*, * *}$ & $1.09(0.05)^{*, * *, \dagger}$ & $1.14(0.04)^{*, * * *, \dagger}$ \\
\hline
\end{tabular}

$\mathrm{ABG}$, arterial blood gas; $\mathrm{Cl}^{-}$, chloride; $\mathrm{HCO}_{3}$, bicarbonate; lonized $\mathrm{Ca}^{2+}$, ionized calcium; $\mathrm{K}^{+}$, potassium; $\mathrm{Mg}$ :creat, magnesium to creatinine ratio; $\mathrm{Na}^{+}$, sodium; Phos:creat, phosphorus to creatinine ratio.

${ }^{*} P<0.05$ for comparisons with controls. ${ }^{* *} P<0.05$ for comparison with low-chloride group. ${ }^{* *} P<0.05$ for comparison with low-sodium/chloride group. ${ }^{\dagger} P<0.05$ for comparison with low-chloride + furosemide group.

Table 3. Clinical and laboratory parameters (M (SD)) in Sprague-Dawley rats by dietary intervention group

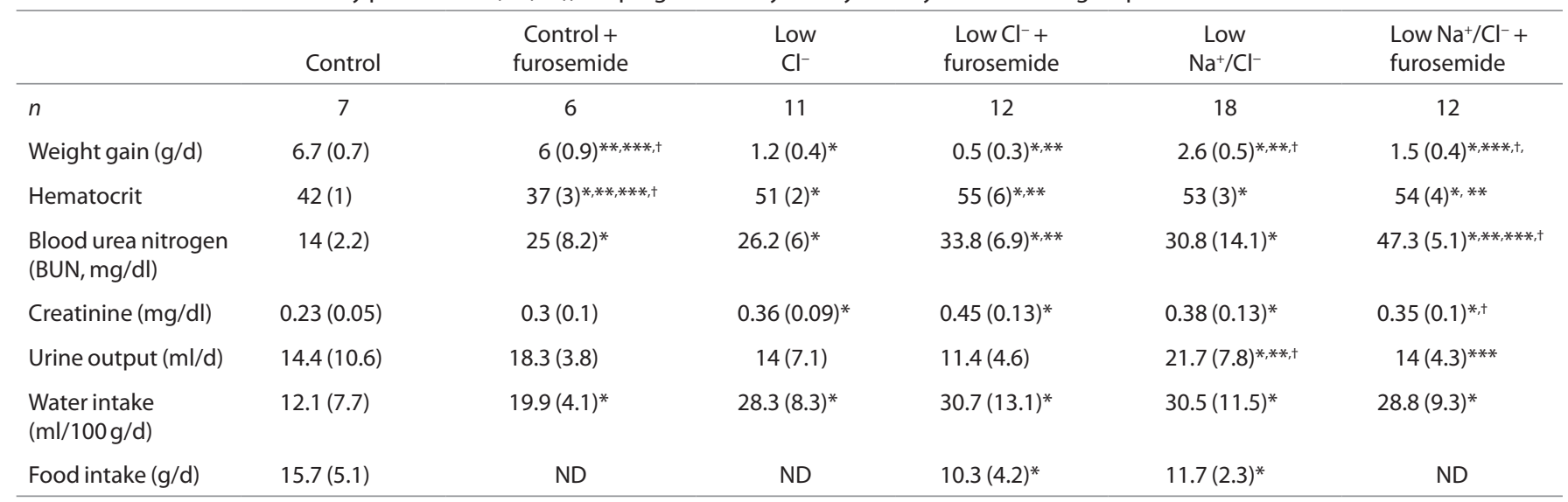

ND, not done.

${ }^{*} P<0.05$ for comparisons with controls. ${ }^{* *} P<0.05$ for comparison with low-chloride group. ${ }^{* * *} P<0.05$ for comparison with low-sodium/chloride group. ${ }^{\dagger} P<0.05$ for comparison with low-chloride + furosemide group.

magnesium excretion occurred among rats fed a low-chloride diet alone, which did not induce NC in the absence of concurrent i.p. furosemide administration.

\section{Effects of Intraperitoneal Furosemide Administration in Dietary Groups}

As shown in Table 2, among rats fed the chloride-deficient diet, i.p. furosemide administration caused a further drop in serum sodium $(P<0.05)$, serum potassium $(P<0.01)$, and serum chloride $(P<0.001)$. This same pattern held true, as expected, for rats fed control diets. In the case of rats fed the control diet, the drop in serum sodium and chloride could be attributed in part to furosemide's effect on increased urinary excretion of sodium $(P<0.001)$ and chloride $(P<0.001)$. However, among rats fed the chloride-deficient diet, there was no significant difference in daily urinary sodium, potassium, or chloride excretion in animals injected with i.p. furosemide. Moreover, i.p. furosemide administration was associated with an increased arterial $\mathrm{pH}$ due to metabolic alkalosis in rats fed the control diet $(P<0.05)$ but not among rats fed the chloride-deficient diet, which had preexisting alkalosis due to hypochloremia with ECF volume contraction. 


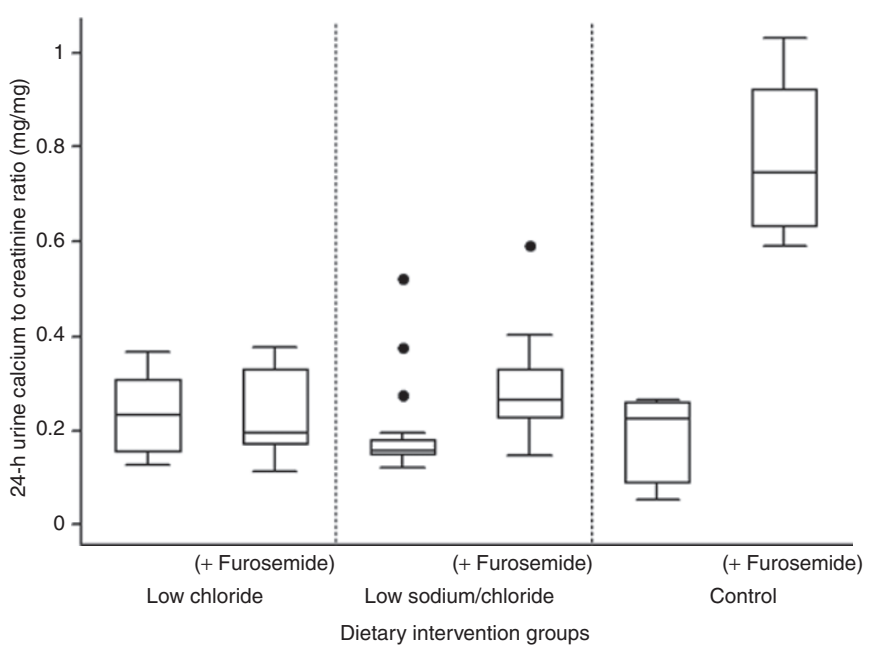

Figure 2. Twenty-four-hour urine calcium excretion categorized by dietary group. Urine calcium excretion expressed as the 24 -h calcium to creatinine ratio $(\mathrm{mg} / \mathrm{mg})$. Solid black dots represent a value $>1.5$ times the interquartile range from the upper quartile value.

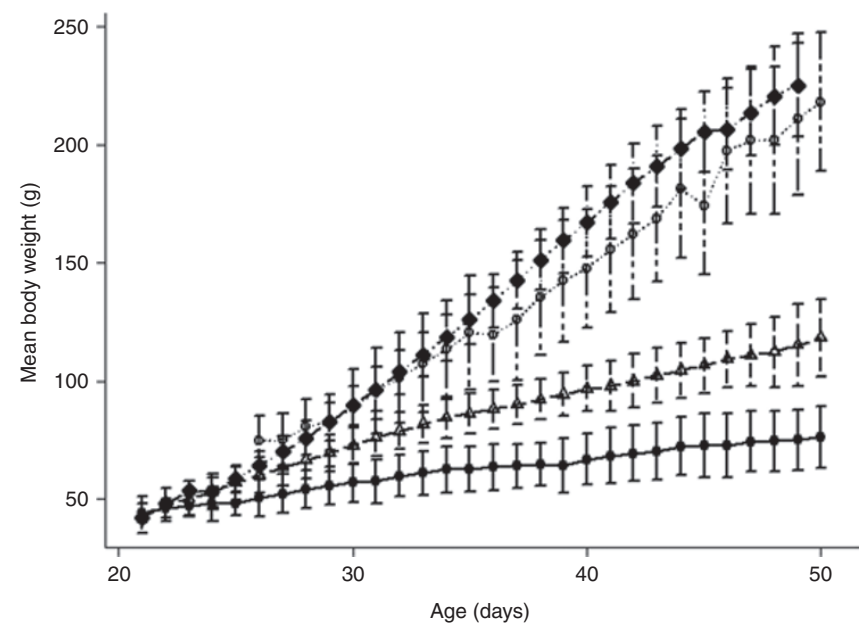

Figure 3. Average daily weight gain by dietary intervention group. Whiskers represent means \pm SD bars. Solid filled black circles with solid line represent rats fed the low-chloride diet. Open triangles with dashed line represent rats fed the low-sodium/chloride diet. Open circles with dotted line represent rats fed a control diet and given i.p. furosemide. Solid black diamonds with dashed line represent rats fed a control diet only.

\section{Blood Pressure Among Rats With and Without NC}

An important negative finding of this study was that there was no difference in systolic, diastolic, or mean blood pressure among rats that did and did not develop NC. As stated above, rats that developed NC had more severe ECF volume contraction as compared with those that did not develop NC. This, however, was not associated with an increased or decreased blood pressure in these animals.

Growth in Rats Fed Chloride or Sodium/Chloride-Deficient Diets As shown in Figure 3, growth (e.g., weight gain) significantly differed among dietary intervention groups $(P<0.001)$, with the chloride-deficient rats having the poorest weight gain followed by rats fed the sodium/chloride-deficient diet in comparison to controls. Rats fed a control diet had normal weight gain. Among rats fed a low-chloride or low-sodium/chloride diet, those injected with furosemide had poorer weight gain than the noninjected animals $(P$ value $<0.05)$. There was no difference in weight gain among rats fed a control diet in the presence or absence of furosemide treatment. At the time of killing (e.g., $7 \mathrm{wk}$ of age), there was a significant difference in length $(13.4 \mathrm{~cm}$ vs. $20.1 \mathrm{~cm}, P<0.001)$ in rats fed the low-chloride vs. the control diet. There was no difference in length between rats fed a lowchloride diet and those receiving a sodium/chloride-depleted diet. Rats from the low-chloride + furosemide and low-sodium/ chloride dietary groups had lower absolute daily voluntary food intake relative to controls (Table 3 ). There was no difference in daily food intake between rats in the low-chloride + furosemide and low-sodium/chloride dietary groups.

\section{DISCUSSION}

The treatment of preterm neonates with furosemide has been associated with the development of NC and renal injury. The mechanisms by which furosemide induces these changes are not clearly understood, and there is a need to develop a reliable small-animal model system to study this process. To address this issue, we developed a rat model of $\mathrm{NC}$ and explored the roles of furosemide, sodium, and chloride depletion in the pathogenesis of NC. Surprisingly, we found that severe chronic dietary sodium and chloride depletion consistently induced NC in young Sprague-Dawley rats not treated with furosemide. These findings were associated with severe ECF volume contraction; hypochloremic, hypokalemic, metabolic alkalosis; and increased urine phosphorus excretion (Table 1). Of interest, we did not detect NC in young rats treated with furosemide and fed a control diet. By contrast, 50\% of the rats treated with furosemide while fed a selective chloride-deficient diet developed NC. Taken together, these findings suggest that the three major trigger mechanisms by which furosemide may precipitate the development of NC in preterm infants are through the induction of ECF volume contraction with hypochloremia, increased urine phosphorus excretion, and HC. However, the potential role of HC could not be confirmed in our animal model system.

There are several potential clinically relevant findings that can be derived from these data. First, this study describes a reliable small-animal model system to study the pathogenesis of $\mathrm{NC}$ in preterm infants. This model produces reliable and severe NC in a significant proportion of rats (50-100\%) (Table 1). Previous studies have shown that weanling rats provide a clinically relevant model system to study how chronic changes in dietary sodium and chloride depletion modulate the ECF fluid volume, total body growth, muscle protein synthesis, and the activation of the systemic and local renin-angiotensin system (21-24). The rapidly growing organisms of preterm infants and young rats need electrolytes to support the expansion of ECF volume required for normal growth (21,25-27). Furthermore, young rats are more susceptible to developing NC as compared with adult rats (28), and they are more sensitive to the effects of dietary sodium/chloride restriction (26). In agreement with previous studies $(21,25)$, our data suggest that the stunted growth seen in rats fed the sodium- and/or 


\section{Articles | Tuchman et al.}

chloride-deficient diets, can be at least partially explained by the reduced caloric intake and severe ECF volume contraction.

Second, our model highlights the importance of both sodium and chloride depletion in addition to volume contraction in the pathogenesis of NC. For example, sodium supplementation has been shown to improve growth and prevent NC in SpragueDawley rats that were treated with i.p. furosemide for $4 \mathrm{wk}$ (27). Furthermore, as previous clinical data also suggest, calcifications do not resolve in all animals after discontinuation of furosemide treatment (17). However, in the present and previous studies, we found that rats fed a selective sodium-deficient diet did not develop NC (21). Our findings provide strong support to the notion that a combination of severe dietary sodium and chloride depletion leading to significant ECF volume contraction may play a key role in the pathogenesis of NC in preterm neonates. For reasons of optimization of lung function, these patients may be subjected to fluid restriction and/or furosemide administration, leading to ECF volume contraction. Hypochloremic metabolic alkalosis is associated with bicarbonaturia and increased urinary sodium and potassium losses. Thus, chronic chloride-depleted rats also become depleted of sodium and potassium. The impact of sodium chloride and volume depletion in the pathogenesis of NC has been demonstrated in children with CLD (15). Nevertheless, it should be noted that the rats fed the selective chloride-deficient diet alone showed severe ECF volume contraction and hypochloremic, hypokalemic, metabolic alkalosis, but did not develop NC. These findings argue very strongly in favor of the hypothesis that other risk factors (e.g., increased urine phosphorus excretion, $\mathrm{HC}$ ), in addition to ECF volume contraction and hypochloremia, are needed to develop NC. Unfortunately, we were unable to generate a definitive conclusion regarding the potential role of $\mathrm{HC}$ in our rat model, given that furosemide did not appear to induce significant $\mathrm{HC}$ in chloride-depleted rats that developed NC (Figure 2). Previous studies detected a similar finding in chloride-depleted adult rats (18). By contrast, increased urine phosphorus excretion appears to be a constant finding in young rats with NC (Table 2). For example, the rats fed the combined sodium- and chloride-restricted diet had the highest proportion (nearly 100\%) and severity of NC as well as the highest phosphorus excretion of all intervention groups (Table 2). Of note, weanling rats fed the selective chloride-depleted diet showed very low values of urinary phosphorus, as compared with all other groups. These findings may be attributable to the lower phosphorus content of the chloride-deficient diet $31 \%$ of that of the control diet), decreased food intake observed in the rats given furosemide that were fed a low-chlorine diet, and the severe ECF volume contraction changes found in these rats, which should further increase the reabsorption of phosphorus in renal proximal tubules. Nevertheless, when assessing the results in the selective chloride-deficient rats treated with i.p. furosemide, we noted that the $50 \%$ of rats that developed NC had a 10-fold higher urinary phosphorus excretion as compared with those that did not develop disease. Prior animal studies have stressed the importance of phosphorus and sulfate loading in the development of $\mathrm{NC}$ with chloride restriction $(18,19)$.
Thus, it is tempting to speculate that the low urinary phosphorus values detected in selective chloride-deficient rats may play a protective role in preventing NC. Alternatively, we did not exclude the possibility that increased phosphaturia detected in all rats that developed NC may be a secondary event, rather than the primary pathogenic factor for NC. In either case, further studies are needed to clarify these important issues.

Previous studies in humans have not consistently revealed a phosphaturic effect of furosemide (29), and the effect in animals has varied depending on the presence of parathyroid hormone (PTH) (30). Although NC in our young rats was associated with higher urinary excretion of phosphorus, it is clear that this was not due to the direct effects of furosemide, given that the highest phosphate excretion occurred in the rats fed with the combined chloride- and sodium-deficient diet that were not treated with furosemide (Table 2). Given that the calcium aggregates seen on histology were in large part composed of calcium phosphate (Figure 1) and the fact that increased phosphaturia seems to be highly associated with the development of NC in our rat model (Table 2), changes in PTH secretion or sensitivity may have an important role in the process as well. Indeed, as shown in Table 2, the two groups with the highest serum bicarbonate concentration had a more significant decrease in serum ionized calcium concentration, which could have stimulated PTH secretion. Furthermore, a previous study showed that PTH serum levels were increased in rats treated with furosemide that developed NC, and that the severity of NC and kidney calcium content was reduced in rats treated with the calcimimetic NPS R-46731 (31). Unlike previous studies (17), we failed to induce NC by injecting i.p. furosemide into young rats subjected to a control diet. The reason for this is not currently understood, but other groups also failed to induce NC in rats injected with furosemide alone (18). Thus, factors in addition to furosemide and PTH should be considered. In any case, this is an important area for future research endeavors.

Studies on NC developing in the context of a high-phosphorus diet or magnesium depletion have not shown regression when animals were switched to control diets $(32,33)$. Whether this is also the case in our animal model is an area requiring further investigation. It is unclear whether NC associated with sodium/ chloride depletion is associated with long-term alterations in renal function. NC induced by excessive dietary phosphorus has been associated with increased urine albumin excretion (34).

Our study has a few limitations. The first is that the kidney calcium content was not measured directly. However, previous studies have shown that kidney calcium content strongly correlates with the severity of NC (35). The second is the lack of longitudinal time course follow-up to determine the minimum amount of time required to develop $\mathrm{NC}$ as well as the initial changes in urinary phosphorus and electrolyte excretion. The third is that without measurement of PTH in our animal model, obtaining a comprehensive understanding of the pathogenesis of NC is limited. Fourth, we could not directly determine the calciuric effect of furosemide within each study group because paired urine specimens were not obtained pre- and postfurosemide administration within each rat. Finally, we used serum 
creatinine, blood urea nitrogen, and hematocrit levels to estimate renal function and ECF volume status. Nevertheless, in previous studies using a similar rat model system, we showed that in the absence of bleeding disorders these markers can be correlated with changes in the renin-angiotensin-aldosterone system and ECF volume contraction (22).

In summary, we have developed a reliable rat model system to study the pathogenesis of NC in preterm infants. We conclude that chronic dietary sodium and chloride depletion leading to severe ECF volume contraction, hypochloremic metabolic alkalosis, and increased phosphaturia constitute a major risk factor for the development of NC in young rats. These findings suggest that furosemide may precipitate the development of NC in preterm infants, at least partially through this simple mechanism. Further studies are needed to validate this hypothesis in preterm infants and to elucidate the basic mechanism involved in the pathogenesis of NC in this new animal model system.

\section{METHODS}

The following protocol was approved by the institutional animal care and use committee of the Children's National Medical Center research institute.

\section{Subjects and Protocol}

Three-week-old, male Sprague-Dawley rats (Taconic, Germantown, NY) were fed diets deficient in sodium $(<0.02 \%)$, chloride $(<0.005 \%)$, or both electrolytes for $4 \mathrm{wk}$ ( $n=12-17$ rats per group). Control rats were fed a standard rodent diet $(0.13 \%$ sodium, $0.54 \%$ potassium, $0.55 \%$ calcium, $0.56 \%$ phosphorus, and $0.03 \%$ sulfate). The sodium- and combined sodium/chloride-deficient diets contained identical amounts of calcium, phosphorus, and sulfate relative to the control diet. As compared with the control diet, the selective chloride-deficient diet had 0.46, 0.31 , and $0.55 \%$ of the calcium, phosphorus, and sulfate content, respectively. The caloric content of all diets was $\sim 4 \mathrm{kcal} / \mathrm{g}(3.86-4.1 \mathrm{kcal} / \mathrm{g})$, with the control, low-sodium, and low-sodium/chloride diets having slightly more calories obtained from protein (casein 20\%) than the low-chloride diet alone (casein 18.3\%). All diets were provided through MP Biomedicals (Solon, OH). Deionized water was administered to all animals. A subgroup of rats from each dietary intervention group was administered $40 \mathrm{mg} / \mathrm{kg}$ of furosemide via daily i.p. injection. Metabolic cages were used to collect 24-h urine specimens and measure daily food intake in subgroups of rats between 6 and $7 \mathrm{wk}$ of age. Animals were killed at $7 \mathrm{wk}$ of age. At the time of euthanasia, animals were anesthetized with either pentobarbital $(35 \mathrm{mg} / \mathrm{kg})$ or ketamine $(80-100 \mathrm{mg} / \mathrm{kg})$ and xylazine $(5-10 \mathrm{mg} / \mathrm{kg})$.

\section{Measurements}

Urine calcium was measured via colorimetric assay $(\mathrm{mg} / \mathrm{dl}$, interassay coefficient of variation $(\mathrm{CV})=0.9 \%)$. Urine creatinine was measured via photoabsorbtiometry utilizing the modified Jaffe reaction $(\mathrm{mg} / \mathrm{dl}$, $\mathrm{CV}=1.1 \%)$. Urine sodium $(\mathrm{mmol} / \mathrm{l}, \mathrm{CV}=3.8 \%)$, potassium $(\mathrm{mmol} / \mathrm{l}$, $\mathrm{CV}=2.6 \%)$, and chloride $(\mathrm{mmol} / \mathrm{l}, \mathrm{CV}=2.4 \%)$ were measured via ionselective electrodes. Daily water intake was measured in all animals while in metabolic cages. Blood pressure and heart rate were measured via indwelling arterial transducer (femoral or carotid access) before the rats were killed. Whole blood was collected in lithium heparin tubes via arterial catheter for electrolytes (sodium, potassium, and chloride), ionized calcium, $\mathrm{pH}$, and bicarbonate. Hematocrit was sampled via arterial line and measured via a hemocytometer.

Kidneys were harvested from all animals and sectioned longitudinally, fixed in $10 \%$ buffered formalin, and stained with both von Kossa (nonspecific calcium) and Alizarin red (calcium phosphate-specific) stains. All renal histology slides were reviewed by study investigators for the presence of calcium deposition in the renal parenchyma. A subset of slides with confirmed NC via von Kossa were stained with hematoxylin and eosin and viewed under polarized light for the presence of calcium oxalate aggregates. NC severity was graded on a 1-5 scale by the study investigators on the basis of the proportion of renal tubular parenchyma with calcium deposition on von Kossa staining. Severe NC was defined as grades $4-5$.

\section{Data Analysis}

A 24-h urinary output was collected for calcium excretion after 4 wk of exposure to each diet and analyzed as $\mathrm{mg}$ calcium per $\mathrm{mg}$ creatinine ( $\mathrm{mg} / \mathrm{mg}$ ). Twenty-four hour urine output was analyzed as $\mathrm{ml} / \mathrm{d}$, and daily fluid intake was converted to $\mathrm{ml} / 100 \mathrm{~g} / \mathrm{d}$ for all analyses. Food intake was analyzed as $\mathrm{g} / \mathrm{d}$. All statistical analyses were performed in STATA 11 (StataCorp, College Station, TX). Results of weight gain and fluid intake, as well as urine and blood measures, were expressed as means \pm SD for each dietary group. One-way ANOVA was used to evaluate differences in serum and urine electrolytes among the eight different groups of rats, and the Students' $t$-test was used to make comparisons between groups. Urine and blood parameters were compared between rats that did and did not develop NC using the $t$-test and then further examined for independent relationships using multivariate logistic regression. Two regression models were constructed for this purpose. The first examined relations between the development of NC (dependent variable) and urine sodium $(\mathrm{mmol} / \mathrm{d})$, potassium $(\mathrm{mmol} / \mathrm{d})$, chloride $(\mathrm{mmol} / \mathrm{d})$, calcium to creatinine ratio $(\mathrm{mg} / \mathrm{mg})$, and urine volume $(\mathrm{ml} / \mathrm{d})$. The second model examined the relationship between $\mathrm{NC}$ and plasma sodium $(\mathrm{mmol} / \mathrm{l})$, potassium $(\mathrm{mmol} / \mathrm{l})$, chloride $(\mathrm{mmol} / \mathrm{l})$, ionized calcium, bicarbonate $(\mathrm{mg} / \mathrm{dl})$, and whole-blood hematocrit $(\%)$. These models were repeated for rats grouped on the basis of the presence of severe NC (NC severity category $>3$ ).

\section{ACKNOWLEDGMENTS}

The authors acknowledge Pedro Jose, director of Pediatric Nephrology Research and professor in the Departments of Pediatrics and Physiology \& Biophysics at the University of Maryland School of Medicine, for his critical advice, expertise, and laboratory support in the performance of this project.

\section{STATEMENT OF FINANCIAL SUPPORT}

This study was supported by the National Institutes of Health $(\mathrm{NIH}$; Bethesda, MD) Child Health Research Career Development Award (05K12-HD00139912) and NIH grants R01 DK-049419 and R01 HL102497.

Disclosure: The authors declare no conflict of interest.

\section{REFERENCES}

1. Pasternack A, Perheentupa J, Launiala K, Hallman N. Kidney biopsy findings in familial chloride diarrhoea. Acta Endocrinol 1967;55:1-9.

2. Hufnagle KG, Khan SN, Penn D, Cacciarelli A, Williams P. Renal calcifications: a complication of long-term furosemide therapy in preterm infants. Pediatrics 1982;70:360-3.

3. Cranefield DJ, Odd DE, Harding JE, Teele RL. High incidence of nephrocalcinosis in extremely preterm infants treated with dexamethasone. Pediatr Radiol 2004;34:138-42.

4. Hein G, Richter D, Manz F, Weitzel D, Kalhoff H. Development of nephrocalcinosis in very low birth weight infants. Pediatr Nephrol 2004;19:616-20.

5. Hoppe B, Duran I, Martin A, et al. Nephrocalcinosis in preterm infants: a single center experience. Pediatr Nephrol 2002;17:264-8.

6. Ketkeaw K, Thaithumyanon P, Punnahitananda S. Nephrocalcinosis in very low birth weight infants: a single center experience. J Med Assoc Thai 2004;87:Suppl 2:S72-7.

7. Schell-Feith EA, Kist-van Holthe JE, Conneman N, et al. Etiology of nephrocalcinosis in preterm neonates: association of nutritional intake and urinary parameters. Kidney Int 2000;58:2102-10.

8. Holmberg C. Congenital chloride diarrhoea. Clin Gastroenterol 1986;15:583-602.

9. Downing GJ, Egelhoff JC, Daily DK, Thomas MK, Alon U. Kidney function in very low birth weight infants with furosemide-related renal calcifications at ages 1 to 2 years. J Pediatr 1992;120(4 Pt 1):599-604.

10. Ezzedeen F, Adelman RD, Ahlfors CE. Renal calcification in preterm infants: pathophysiology and long-term sequelae. J Pediatr 1988;113:532-9.

11. Holmberg C, Perheentupa J, Pasternack A. The renal lesion in congenital chloride diarrhea. J Pediatr 1977;91:738-43. 
12. Bérard E, Dageville C, Bekri S, Boutté P, Coussement A, Mariani R. Nephrocalcinosis and prematurity: importance of urate and oxalate excretion. Nephron 1995;69:237-41.

13. Woolfield N, Haslam R, Le Quesne G, Chambers HM, Hogg R, Jureidini $\mathrm{K}$. Ultrasound diagnosis of nephrocalcinosis in preterm infants. Arch Dis Child 1988;63:86-8.

14. Pope JC 4th, Trusler LA, Klein AM, Walsh WF, Yared A, Brock JW, 3rd. The natural history of nephrocalcinosis in premature infants treated with loop diuretics. J Urol 1996;156:709-12.

15. Wedenoja S, Ormälä T, Berg UB, et al. The impact of sodium chloride and volume depletion in the chronic kidney disease of congenital chloride diarrhea. Kidney Int 2008;74:1085-93.

16. Campfield T, Braden G, Flynn-Valone P, Powell S. Effect of diuretics on urinary oxalate, calcium, and sodium excretion in very low birth weight infants. Pediatrics 1997;99:814-8.

17. Alon US, Kaplan RA, Gratny LL, Nichols MA. Histological long-term outcome of furosemide-induced nephrocalcinosis in the young rat. Pediatr Nephrol 1996;10:191-4.

18. Levine DZ, Roy D, Tolnai G, Nash L, Shah BG. Chloride depletion and nephrocalcinosis. Am J Physiol 1974;227:878-83.

19. Holliday MA, Bright NH, Schulz D, Oliver J. The renal lesions of electrolyte imbalance. III. The effect of acute chloride depletion and alkalosis on the renal cortex. J Exp Med 1961;113:971-80.

20. Mackay EM, Oliver J. Renal damage following the ingestion of a diet containing an excess of inorganic phosphate. J Exp Med 1935;61:319-34.

21. Ray PE, Lyon RC, Ruley EJ, Holliday MA. Sodium or chloride deficiency lowers muscle intracellular $\mathrm{pH}$ in growing rats. Pediatr Nephrol 1996;10:33-7.

22. Ray PE, Castrén E, Ruley EJ, Saavedra JM. Different effects of sodium or chloride depletion on angiotensin II receptors in rats. Am J Physiol 1990;258(4 Pt 2):R1008-15.

23. Lane PH, Tyler LD, Schmitz PG. Chronic administration of furosemide augments renal weight and glomerular capillary pressure in normal rats. Am J Physiol 1998;275(2 Pt 2):F230-4.
24. Lane PH. Furosemide treatment, angiotensin II, and renal growth and development in the rat. Pediatr Res 1995;37:747-54.

25. Heinly MM, Wassner SJ. The effect of isolated chloride depletion on growth and protein turnover in young rats. Pediatr Nephrol 1994;8:55560.

26. Orent-Keiles E, Robinson A, McCollum EV. The effects of sodium deprivation on the animals organism. Am J Physiol 1937;119:651-61.

27. Gratny LL, Ringer K, Hall RT, Alon US. Salt supplementation, growth, and nephrocalcinosis in the furosemide-treated weanling rat. Biol Neonate 1997;71:37-45.

28. Hitchman AJ, Hasany SA, Hitchman A, Harrison JE, Tam C. Phosphateinduced renal calcification in the rat. Can J Physiol Pharmacol 1979;57: 92-7.

29. McNabb WR, Noormohamed FH, Brooks BA, Lant AF. Renal actions of piretanide and three other "loop" diuretics. Clin Pharmacol Ther 1984;35:328-37.

30. Haas JA, Larson MV, Marchand GR, Lang FC, Greger RF, Knox FG. Phosphaturic effect of furosemide: role of PTH and carbonic anhydrase. Am J Physiol 1977;232:F105-10.

31. Pattaragarn A, Fox J, Alon US. Effect of the calcimimetic NPS R-467 on furosemide-induced nephrocalcinosis in the young rat. Kidney Int 2004;65:1684-9.

32. Soeterboek SJ, Ritskes-Hoitinga J, Lemmens AG, Beynen AC. Phosphorusinduced nephrocalcinosis in female rats: a study on regression and clinical abnormalities. Lab Anim 1991;25:258-62.

33. Heaton FW, Anderson CK. The mechanism of renal calcification induced by magnesium deficiency in the rat. Clin Sci 1965;28:99-106.

34. Van Camp I, Ritskes-Hoitinga J, Lemmens AG, Beynen AC. Diet-induced nephrocalcinosis and urinary excretion of albumin in female rats. Lab Anim 1990;24:137-41.

35. Hoek AC, Lemmens AG, Mullink JW, Beynen AC. Influence of dietary calcium:phosphorus ratio on mineral excretion and nephrocalcinosis in female rats. J Nutr 1988;118:1210-6. 